\title{
PERSPECTIVE
}

\section{FinAl ThOUghts}

My term as president is drawing to a close and in sitting down to write this last Perspective I began to reflect on the events of the past year - our successes, our challenges and our path for the future.

Over the course of the past year I have, through my previous Perspectives, attempted to address the "where," "how","who," "what" and "why" questions facing CIF/IFC leaving the "when" for this final column.

Our ability as an organization to move forward is tied directly to our ability to address and resolve the challenges we face. If we accept the premise that any situation can be broken down into its elemental stages, then it follows that we can support the construct of laying a solid foundation to promote a given issue, give it fair address and bring about conclusion whenever possible. I believe that the focus this year has been on answering the questions in a fashion that enables a strong series of actions from this time forward.

I must say that often organizations are paralyzed by complexity and unwilling to take risks to move away from the known into the unfamiliar where action can take place. Rear Admiral Grace Hopper once said the most dangerous phrase in any organization is "it's always been done that way." I agree wholeheartedly. For progress to occur organizations need to take risks and must challenge the membership to move forward.

Together we have looked at the big picture, recognized that we are not alone in that picture and hopefully have figured out how to bring others and ourselves into focus within the mural. We have taken the time to evaluate how to improve communication to allow various players to contribute and be effective. The essentials of our communication — our values, attitudes and beliefs — are geared to ensure our messages are as complete as possible. Finally, we determined that each and every one of us has a role to play in the delivery of these messages, whether they are expressed locally, nationally or internationally.

We continue to challenge ourselves with complex issues because we believe CIF/IFC can help the forest community exist in a competitive and complex landscape. The values of forests, however defined, are affected and affect components of issues such as climate change, biological diversity, endangered species, protected areas, desertification, social wellbeing, community maintenance, institutional frameworks and consumer preferences.

By spending this year addressing how we do business and working on the banner issue of stewardship through partnership we are now positioned to tackle effectively the external issues with a stronger, more focussed organization.

I would like to think that future presidents will be able to tackle the many and varied issues facing forestry secure in the knowledge they are working from the solid foundation $>\mathbf{5 6 7}$

\section{DERNIERES REFLEXIONS}

Mon terme de présidente s'achève alors que je suis à rédiger cette dernière perspective que j'ai voulu être un reflet des événements de l'année dernière, nos succès, nos défis et notre direction vers l'avenir.

Au cours de la dernière année, j'ai tenté, au moyen de mes perspectives précédentes, de répondre aux questions relatives aux "où", "comment", "qui", "quoi" et "pourquoi" qui touchent l'Institut, gardant le "quand" pour cette dernière chronique.

Notre capacité en tant qu'organisation d'aller de l'avant est directement reliée à notre capacité de répondre et de résoudre les défis qui nous font face. Si nous acceptons la prémisse que toute situation peut être subdivisée en ses éléments de base, il s'en suit donc que nous pouvons supporter la construction d'une solide fondation afin de promouvoir n'importe quel enjeu, $y$ faire face adéquatement et apporter une conclusion dans tous les cas possibles. Je crois que le point central de nos actions cette année a été de répondre aux questions d'une manière qui nous a permis d'entreprendre une longue série d'actions concrètes.

Je dois dire que bien souvent les organisations sont paralysées par la complexité et le manque de désir à prendre des risques afin de s'éloigner de ce qui est familier pour se diriger vers l'inconnu engendré par l'action. Le contre-amiral Grace Hopper a dit un jour que la période la plus dangereuse pour toute organisation est
de dire "que cela a toujours été comme ça". Je suis entièrement d'accord. Pour être en mesure de progresser, les organisations doivent prendre des risques et doivent inciter leurs membres à aller de l'avant.

Ensemble, nous avons examiné le contexte général, et reconnu que nous ne sommes pas seuls dans ce portrait et qu'heureusement nous avons figuré comment il possible d'amener les autres ainsi que nous-mêmes à se rattacher au contexte. Nous avons pris le temps d'évaluer comment améliorer les communications afin de permettre à différents acteurs d'apporter leurs contributions et d'être efficaces. Les éléments essentiels de nos communications - nos valeurs, attitudes et croyances - sont réunis pour s'assurer que nos messages sont le plus complet possible. Finalement, nous avons déterminé que tous et chacun d'entre nous avons un rôle à jouer dans la divulgation de nos messages, que ce soit sur une scène locale, nationale ou internationale.

Nous continuons de nous mettre au défi au moyen d'enjeux complexes parce que nous croyons que l'Institut peut aider la communauté forestière à vivre dans un milieu compétitif et complexe. Les valeurs rattachées aux forêts, peu importe comment elles sont définies, sont affectées et affectent les composantes des enjeux comme le changement climatique, la biodiversité biologique, les espèces menacées, les territoires protégés, la désertification, le mieux-être de la société, le maintien $\mathbf{\$ 5 6 7}$ 
planning, to address, where possible, local issues and concerns, and to participate in regular reviews of their performance. Nationally, it was the industry itself that proposed the idea of sustainable forestry certification - and, in Ontario, it was the industry that first suggested public advisory committees as a way for companies and communities to develop closer, and therefore more effective, working relationships.

We need the support of our communities. We need those people whose livelihoods are dependent on the industry to be vocal on our behalf - not because we say they should, but because they understand the way we work and why; because they're involved in forest management planning; because they see not only harvesting, but the subsequent regeneration; and because we have clearly demonstrated the lasting benefits for everyone concerned.

When we think about sustainable development, forestry and communities, the tendency is to consider the industry as a whole. And, through my experience as president of an industry association, I do believe that we can accomplish far greater things collectively than we can in isolation. There is no question that we need a cohesive global framework that facilitates action on a country-by-country basis. But at the end of the day, sustainability can only be achieved - or not - one community at a time.

R. Marie Rauter is President of the Ontario Forest Industries Association.

\section{WITHOUT FEAR or FAVOUR: culling \& scaling timber in Canada 1762-1992.}

a study on the evolution of culling and scaling practice in each Canadian province; it's also about sharp practices, plagiarism, political interference $\&$ bureaucratic ineptitude.

30 appendices, 15 figures, 13 tables, index, $225 \mathrm{p}$ $\$ 37.40, \$ 30.00$ USA \& overseas, includes tax \& mailing

A great gift or reference 3 ways to order

T.G. Honer, 3835 Haro Road, Victoria, BC, V8N 4 A6

Fax: (250) 721-1029; email: honertg@islandnet.com ments de la communauté forestière. Depuis ce moment, plusieurs des engagements pris dans le Code sont devenus des exigences légales. Par exemple, l'obligation d'inclure la participation du public dans le processus de planification d'aménagement forestier; de tenir compte, lorsque possible, des enjeux et des intérêts locaux; et d'entreprendre des révisions régulières de la performance. À l'échelle nationale, l'industrie a proposé d'elle-même l'idée de la certification de la foresterie durable, et, en Ontario, c'est l'industrie qui a suggéré la première la mise en place de comités consultatifs publics en tant que moyen pour les compagnies et les communautés de développer des relations de travail plus rapprochées, et par le fait même, plus efficaces.

Nous devons appuyer nos communautés. Nous avons besoins des personnes dont le mode de vie dépend de l'industrie pour qu'elles se fassent entendre, non pas parce que nous disons qu'elles le doivent, mais parce qu'elles comprennent notre façon de travailler et pourquoi nous travaillons de cette façon; parce qu'elles sont impliquées dans le processus de planification de l'aménagement forestier; parce qu'elles voient non seulement la récolte, mais également la régénération qui suit; et parce que nous avons clairement fait état des bénéfices durables pour toute personne concernée.

Lorsque nous pensons au développement durable, à la foresterie et aux communautés, nous avons tendance à considérer l'industrie comme un tout. Et, de part mon expérience à titre de présidente d'une association de l'industrie, j'estime que nous avons réalisé beaucoup plus de choses ensemble que nous aurions pu individuellement. Il va s'en dire que nous avons besoin d'un cadre de travail cohésif qui facilite la pose de geste concret sur une base de pays à pays. Mais en bout de ligne, la durabilité ne peut être atteinte, ou pas, qu'une communauté à la fois.

\section{R. Marie Rauter est présidente de l'Association des industries forestières de l'Ontario}

Perspective des communautés, les cadres institutionnels et les préférences des consommateurs.

En ayantconsacrer cette année à étudier comment nous pouvons faire affaire et travailler sur la thématique annuelle de l'intendance par l'entremise du partenariat, nous sommes maintenant en position pour affronter des enjeux externes puisque que notre organisation est plus forte et mieux centrée.

J'aimerais penser que les prochains présidents seront en mesure de faire face aux nombreux et variés enjeux qui touchent la foresterie, sachant qu'ils sont rassurés de travailler sur une base solide construite cette dernière année que nous avons consacrée à reconfirmer et à renforcer le plus grand nombre possible de nos assises.

Je vous remercie de m'avoir donner la possibilité d'être votre présidente et d'avoir contribuer à notre progrès collectif sur plusieurs fronts.

Perspective built this past year as we endeavored to reconfirm and strengthen as many of our foundation blocks as possible.

Thank you for the opportunity to be your president and to contribute to our collective progress on many fronts. 